\title{
FOXP3 and HER-2/ErbB2 in Breast Cancer: Finding Regulatory Links
}

\author{
Abdulrazzaq N. Zghair ${ }^{1}$, Nihad Khalawe Tektook ${ }^{2}$, Ahmed A. Mohsin ${ }^{1}$ \\ ${ }^{1}$ Lect., ${ }^{2}$ Assist Prof., Middle Technical University/College of Medical \& Health Technology, \\ Medical Laboratory Techniques Dep. Iraq
}

\begin{abstract}
The transmembrane tyrosine kinase receptor, HER2/ErbB2, has been a subject of many studies owing to its predictive and prognostic values and its being a target of antibody-mediated treatment. Retrospective evidence strongly proposes that the over expression of HER2 is related to reduced disease free and overall survival in node positive and perhaps also node negative breast cancers. Prospective attempts showed that antibodies to HER 2 can form tumor responses in advanced breast cancer women which overexpress this molecule. The existence of forkhead box protein 3 (FOXP3) in cells of breast cancer is a subject of debate. We have systematically analyzed the FOXP3 expression in 20 breast carcinoma samples at transcript levels. Recent progresses in understanding breast cancer crosstalk, homing processes, tumor cell dormancy, premetastatic niche formation and finally recognition of their micromilieu cytokines, growth factors and chemokinesmight provide the foundation of developing targeted treatment plans potentially rendering primary carcinomas and their metastases more responsive to chemotherapies. The current review has focused on the deep connections between HER-2 and FoxP3 in context of considering the determination of both of these molecules together to rationalize or more personalize the future research directions leading to better treatment for women with breast carcinoma.
\end{abstract}

Keywords: Her-2, Breast cancer, Foxp3, Inhibit, Therapy, Trastuzamab.

\section{Introduction}

Breast cancer is a major cause of concern in women worldwide. It is responsible for an appreciable amount of morbidity and mortality in women, with every $8^{\text {th }}$ women showing symptoms which sooner or later progresses into disease. Human epidermal growth factor receptor 2-overexpressing $\left(\mathrm{Her}-2^{+}\right)$breast Ca represents about $20-25 \%$ of breast cancer and has been shown to be more aggressive as compared to the Her2 ${ }^{-[1-2]}$. Her2 is a reliable biomarker whose overexpression is frequently correlated to poor prognosis and clinical outcomes. Trastuzumabor (Herceptin), the monoclonal antibody which targets the extracellular domain of HER2, has been long used in treatingpatients with Her2+ breast carcinoma. Nevertheless, many patients suffering fromHer $2^{+}$tumors possess de novo resistance without response to Trastuzamab, while others developed resistance after a period of Trastuzamab therapy. Her2 ${ }^{+}$ signaling mechanism has been extensively studied to identify additional targets to design effective treatments to $\mathrm{Her}^{+}$breast cancer patientswith Trastuzumab resistance. One of such potential targets can be Foxp3 which is an important factor overexpressed in all cancers including breast cancer. Its relation to Her2+ signaling shows that it plays an important role in suppressing Her 2 oncogene. On the contrary, Foxp 3 is also a fundamental partner of Treg cells which suppresses the immune response against tumor in tumor milieu. Studies have also shown that Foxp3 inhibition in $\mathrm{Her}^{+}$over expressing breast carcinomas could be correlated with better prognosis and relapse free with general survival ${ }^{[3]}$. Thus, Foxp 3 can be envisaged as a pivotal target whose down regulation in tandem with other breast cancer targeted therapies can have astounding effects in women with breast carcinoma. FOXP3 and related mechanism have an essential role in breast cancer metastasis inhibition and such finding might introduce a new target to clinically control the metastasis of breast cancer in the 
future. There is an inverserelation between expression of FOXP3 nuclei of breast tumor cellsand breast cancer metastasis. Thus, targeting Foxp $3^{+}$Treg cells in the microenvironment of cancer combined with anti-Her2 treatment can be a novel strategy against breast cancer particularly chemotherapy-resistant breast cancers. In this review, we have discussed the relation between Her2 and Foxp3 and their role in breast cancer with some recent therapies targeted against them.

HER2 in Breast Cancer: Her2 is apro oncogene is a member in the family of human epidermal growth factor.Other members of this family are HER-1,HER-3, HER-4 and HER-2 which are involved in more than $1 / 4$ of breast cancer cells ${ }^{[4]} \cdot \mathrm{It}$ is present on the long arm of the chromosome No. 17 and it encodes atransmembrane tyrosine kinase receptor. The Her 2 and the other member receptors have an extracellular ligand binding site that is rich in cysteine residues, a transmembrane domain and an intracellular domain having tyrosine kinase activity. HER2 has no direct ligand.

HER2 is normally present as a monomeric receptoron the surface of epithelial cells of mammary glands at a very low level. In tumor cells, HER2 expression increases manifolds. Studies have shown that gene amplification is the reason behind HER2 overexpression. HER2 receptor is activated by ligand binding followed by dimerisation of receptor throughhomomeric orheteromeric binding with other EFGR receptors. Extracellular domain rich in tyrosine residues is involved in this binding. HER. Neuregulin-1, a cell adhesion molecule, is a direct ligand for HER3 and HER4and binds with either HER3 or HER4. After ligand binding, HER2 forms heterodimer with HER4 or HER3. This dimerisation of receptor is followed by the autophosphorylation of the tyr residues

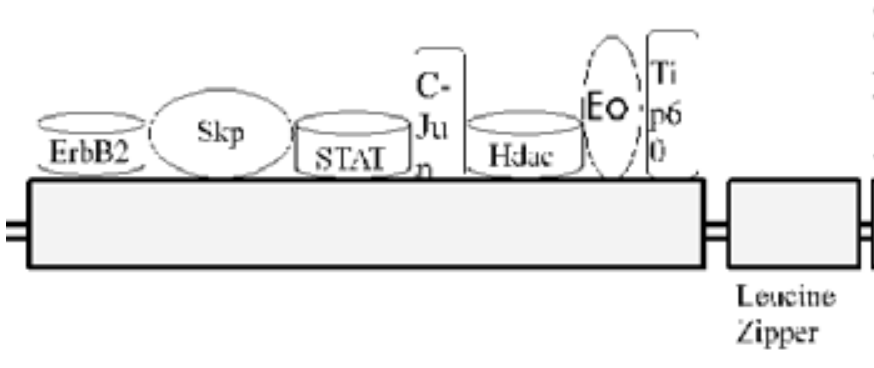

of intracellular domain through the activation of tyrosine kinase activity of HER2, which turns up cascades of events that are involved in the activation of intracellular signaling pathway. Several signal transducers bind to the activated heterodimer through their src homology domain and initiate signal transducing pathways thereby, stimulating PI3/AKt or MAPK signaling pathways andvarious transcription factors and overproduction of VEGF. Various protooncogenes e.g c-fos, c-jun, c-mycetc are also activated.HER-2 over-expression thus activates multiple pathways involved in the process of metastasis ${ }^{[5]}$. The heterodimer also degrades cell cycle inhibitor P27. Loss of P27 causes proliferation of cancer cell[6].

FoxP3 and Immunosuppressive mechanisms in tumor milieu: Forkhead (FKH) box proteins is a bigtranscription factor familyutilized in different cellular processes. The gene encoding (FOXP3) is present on the chromosome Xwhich containseleven coding exons and three non coding exons [25]. The characteristic feature of these transcription factors is the existence of a highly preserved(100) amino acids C- terminal FKH binding domains. The FKH with crystal structure domains showed that it has the DNA binding ability and described as a "wing-helix" owing to its similarity to the butterfly morphology. This highly conserved carboxy terminal FKH domain (a.a.338to421) is responsible for DNA binding ability. The N-termnal domain of FOXP3 comprising of 2 proline-rich regions implicated to mediate transcriptional repressions. The central region of Foxp3 protein comprises a zinc finger (A.A. 200223) \& a leucine zipper (LZ)-like motif (A.A. 240-261), thatpromotesFOXP3 homo-dimer or tetramer formation (Fig. 1) $^{[26] \text {. }}$

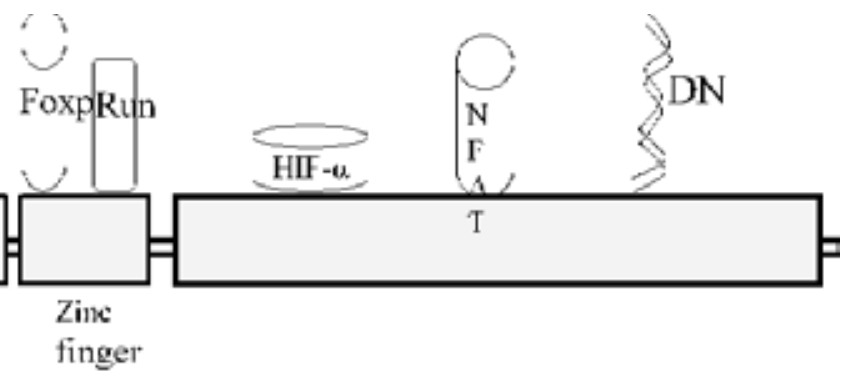

Dimerisation

Domain
Forkleas Domain

Figure 1

Figure (1): Foxp3 domains (a) N- terminal repressor domain having tumor suppressor activity inhibiting the suppression of ErbB2 (erb-b2 receptor tyrosine kinase-2),
SKP2 (S-phase kinase associated protein-2), STAT3 (Signal transducer and activator of transcription 3), c-Jun (transcription factor c-Jun), HDAC7 (histone deacetylase 
7), Eos (Ikaros Family Zinc Finger 4 IKFZ-4), TIP60 (Histone Acetyl TransferaseTIP60). (b) Intermediate domain or dimerization domain comprising of leucine zipper and zinc finger responsible for dimerization of Foxp3 interacts with Foxp3 and Runx1 (runt-related transcription factor 1 ). (c) Forkhead Domain which has DNA binding ability and interact with factors such as NFAT (Nuclear Factor Activated T-cells), the DNA or HIF-1 $\alpha$ (Hypoxia-induciblefactor $1 \alpha)$

The scurfy mutant mouse strain having frame shift mutation of (FKH) box transcription factor (Foxp3)sustainoing a lethal lymphoproliferative disorderscausing death within an age of 3to4 weeks. Mutations in FOXP3 resulted in various autoimmune diseases such as hepatomegaly, splenomegaly, insulitis and massive lymphatic infiltration in liver and skin. Humans (FOXP3)mutationleads to an autoimmune syndrome called IPEX (immune dysregulation, polyendocrinopathyenteropathy and X-linked disease), which is an X-linked immunodeficiency specified as insulin-dependent Diabetes mellitus (IDDM), thyroiditis, massive T-cell infiltrates in several organs accompanied by chronic wastings ${ }^{[27-28]}$.

T reg-mediated suppression mechanisms: Foxp3+ CD25+CD4+ Treg (Foxp3+ Treg cells)cells mediated immune suppression is a primary mechanism in immunological self-tolerance, preventing autoimmune disease development.Treg cell development is impaired by gene mutation that encode Treg Specific transcription factor (foxp3)leading to a lethal multiple organ autoimmune diseasetermed as immuno-dysregulation, poly endocrinopathy, enteropathicand X-linked (I.P.E.X) syndrome. (Foxp-3+Treg) cell depletion by differenttechniquesresults inthe same autoimmune diseases in other normal rodent ${ }^{[29]}$.Tumor tissuesof different cancers including breast are often infiltrated by huge numbers of Treg cells and this abundance of cells is usually implicated in poor clinical prognosis. The removalof (Treg) cells by implying the celldepletion anti CD25 antibodies, by the in vivoantibody administrations histocompatible T-cell-deficient mice can efficientlyeliminate different inoculated syngeneic cancers ${ }^{[30]}$. There was an elevatedcancer infiltrated CD8+ T cells showing a strong activity to kill tumor specific, and in response to rechallengingagainst the same cancercell, caused aquicker anti-tumor response, demonstrating the existence of cancer specificimmunity ${ }^{[31]}$ A number of studies established that Treg cells removal caninduce effecient anti cancer immunity by eliminating the immunologic un-responsivenessto syngeneic cancers. Moreover, among different Treg dependent suppression mechanism, those which are essential to maintain self tolerance have the greatest effect on tumor's immunity. There are onlysmall number of molecules whose expressions are adjusted by direct or indirect Foxp3 and whose deficiencyeliminates Treg suppression functioning leading to serious autoimmunitydisorders. This candidate includes Interleukins 10, 2 and IL-2 receptors subunit, Tfg-ßand C.T.L.A-4. The basicproperty of Treg cell is thehigh affinity IL-2 receptor expressions consisted of CD25, 122 and132 at highly levels, but with rare production of Interleukin-2. Thus, there is a highly dependence of Treg cellson the exogenous Interlukine-2, which is primarilyreleased by the activatedT-conv cell, for itsproliferation and survival.

IL-2 is indispensable for the function and survival of Treg cells, evident by the fact that anti-IL-2 antibodies compromise Treg survival and function, resulting in severe autoimmune disorders as produced by Treg deficiency. Foxp3combines with and attenuates NFAT and AML1 transcription factors which are necessary for IL-2 production, thereby repressesIL-2 production ${ }^{[32-33]}$. Owing to high dependence of Treg cells on IL-2 and self insufficiency in its production, they absorb IL-2 from the surrounding. This limits IL-2 amount which is available for Tconv cells and as a result, leading to the activation and proliferation suppression of the Tconv cells which are essential forimmune responses ${ }^{[34]}$

C.T.L.A-4 is a high effective co-inhibition molecules that is expressed by (Treg) cell and (Tconv) cell following their activations. The C.T.L.A-4Treg-specific deletion in micecauses systemic high multiplication of (Tconv) cell and lethal autoimmunity disorders and affectseveral bodytissue and organs ${ }^{[35]} \cdot A$ heterozygous CTLA-4 mutation in humans is characterized bymany autoimmune symptom accompanied with Treg cells suppressive function's impairment ${ }^{[36]}$. CTLA-4 exhibit a high affinitiesthan (CD28)for their (CD80) and (CD86) ligands.The C.T.L.A-4 which is expressed bythe Treg cell will physically outcompete the (CD28)expressing (Tconv) cellto bindthe (CD80/CD86) on the antigen presentingcell, therefore inhibitsthecostimulation ofTconv cell ${ }^{[37] \cdot}$ The C.T.L.A-4 on Tregcells also down modulatethe (CD80/CD86)expression on the DC,hence, inhibiting the Tconv cells activations ${ }^{[38-39]}$. The C.T.L.A-4 and other accessory molecules are further upregulated by the TCR stimulation of Treg cells, specially the adhesion molecules like LFA-1, whose 
deficiency compromise suppressive activity. Thehigh L.F.A-1 and other accessorymoleculeexpression pribeforeor T.C.R stimulationscanlead toestablish a less threshold to T.C.R-induced activations ofthe Treg cell ${ }^{[40]}$. The attenuated T.C.R signal may savethe Treg cell from activations induced cell deaths upon their exposuresto antigen, assisting them for better survival than(Tconv) cell,since such Treg cells overrideTconvattumor sites. This combined effect of collective highly CD25 \& C.T.L.A-4 expressions, relianceupon the exogenous Interleukine-2 and T.C.Rstimulations havedecisive role in the Treg mediated suppressions.

Foxp3 and cancer: A largenumber of Treg cells often infiltrate different mice \& humantumors. In humans, the lung ${ }^{[41]}$, liver ${ }^{[42]}$, head and neck [43], breast ${ }^{[44]}$, gastrointestinaltract ${ }^{[45]}$ and ovary ${ }^{[46]}$ tumors were shown to bear a highly numbers of tumor infiltrated Treg cell ${ }^{[47]}$. The decreased ratioof tumor infiltrating (CD8+) T cells to FOXP3+ Treg cellswere correlated with bad prognosis, mainly in breast cancer patients $^{[48]}$, gastric ${ }^{[49]}$, and ovarian cancer ${ }^{[50]} \cdot \mathrm{A}$ previous met a-analysis published data specified that in most breast, kidneys, cervix tumors and melanoma, the highly frequent tumor-infiltrated FOXP3+ cell was associated with patient's survival negatively ${ }^{[51]}$.

Interplay between HER2 and FoxP3 in breast cancer: A study by Parejet al on the correlation between circulating Treg cells in Her2 ${ }^{+}$and Her2-Bc cells showed that Her2 $2^{+}$tumors were characterized by increased levels of FoxpTreg cells in the blood of BC patients ${ }^{[52]}$. The high levels of Treg cells have been frequently correlated with $\mathrm{MAB}$ or $\mathrm{LABC}$. Her $2^{+}$and Her2- tumors when subjected to chemotherapy with trastuzumab alone or in combination with other drugs caused overall reduction in the Treg cells frequencies to normal levels. Some of the Her2+ patients initially responding to trastuzumab therapy exhibited disease reoccurrence after sometime which was correlated with increase in Treg cell frequency. A correlation between changes in circulating Treg frequency and plasma HERECD(extracellular domain) implied that at least some of those cells canrespond to and recognize the systemically circulating (HER) proteins. Patients who respond to trastuzumab treatment by decreasing plasma HER-ECD also demonstratedlow frequency of Treg.It was noticed that HER was eliminated from blood circulation by antigen-trastuzumab complex production and uptake by phagocytic cells via combining with FcgR ${ }^{\text {[53-54]. HER- }}$ trastuzumab complex formation also led to maturation, activation and reinforced antigen cross presentation by the Antigen Presenting Cell ${ }^{[55]}$, and along with low Treg frequency, may possibly induce an enhanced antitumor responses.

Foxp3 isrecognized during Scurfin position cloning, which isthe gene responsible for $\mathrm{X}$ linked autoimmunesdisorders in humans and mice (Immune dysregulation, enterophathy, polyendopathy, X-linked \& IPEX) ${ }^{[56-59]}$. Mice heterozygous forFoxp $3^{\text {sf }}$ spontaneously developed malignant tumors among which more than half were mammary carcinomas. To establish a link between mammary carcinomas and Foxp3 mutation mice, heterozygous for Foxp $3^{\text {sf }}$ were treated with a carcinogen, 7,12-dimethylbenz [a] anthracene (DMBA). It was observed that mutation for Foxp $3^{s f}$ but not for $O t c^{s p f}$ gene leads tomainelevation in susceptibility to the mammary carcinomas. A comparison of Foxp3 expression normal and mammary epithelium from bothwild-type and Foxp $3^{\text {sf }+}$ mice showed that Foxp3 mRNA was identified in normal epithelium of both WT and Foxp $3^{\text {sf }}$ mice, but not in mammary carcinomas. This decrease in Foxp3 MRNA was concurrent with significant increase in HER2 mRNA in mammary epithelium. There was also increased HER2 mRNA expression in FOXp $3^{\text {sf/spf }}$ epithelium than in wild type female mice indicating a potential gene dosage impact of in vivo Foxp3 of HER2 regulation. Repression of HER 2 by Foxp 3 is mediated by a direct binding between Forkhead domain of foxp3 onto the promoter region of HER2.The Foxp3 binding site deletion increased the Her 2 promoter activities and relieved Fox p3 mediated Her2 repression. In mostbreast cancers, $\mathrm{LOH}$ alone was enoughfor the locus inactivation, probablyowing to Xchromosomeinactivation. Silencing of Foxp3 gene by Foxp 3 siRNA decreased Foxp 3 expression by more than (100)fold, while increasing Her 2 mRNA by 7 folds. A comparison of Foxp3+ and Foxp3- cancer samples revealed that Foxp $3^{+}$samples has reduced HER2 scores compared with Foxp3- ${ }^{-}$specimens suggesting a decisive role for Foxp3 in repressing HER2 expression.Further results revealed that mice heterozygous toFoxp 3 mutations spontaneously developed high rates of mammary cancer. Cells where WT allele was silenced by $\mathrm{X}$ inactivations had inactive Foxp 3 and overexpression of Her2.Most of these mutations concentrated onzing finger \& FKH domains which inactivated tumor growth inhibition \& repressor activity of Foxp3 (Fig. 2). It could be concluded that the FKH and Zinc finger domains of intracellular transcription factor foxp3 is directly 
boundwith the promoter's region of Her2 and acts as a restraint on the transcription of her 2. Any deletion or mutation in foxp3 relieves Her 2 of this restraince

resulting in its overexpression ultimately causing tumorigenesis ${ }^{[60] .}$

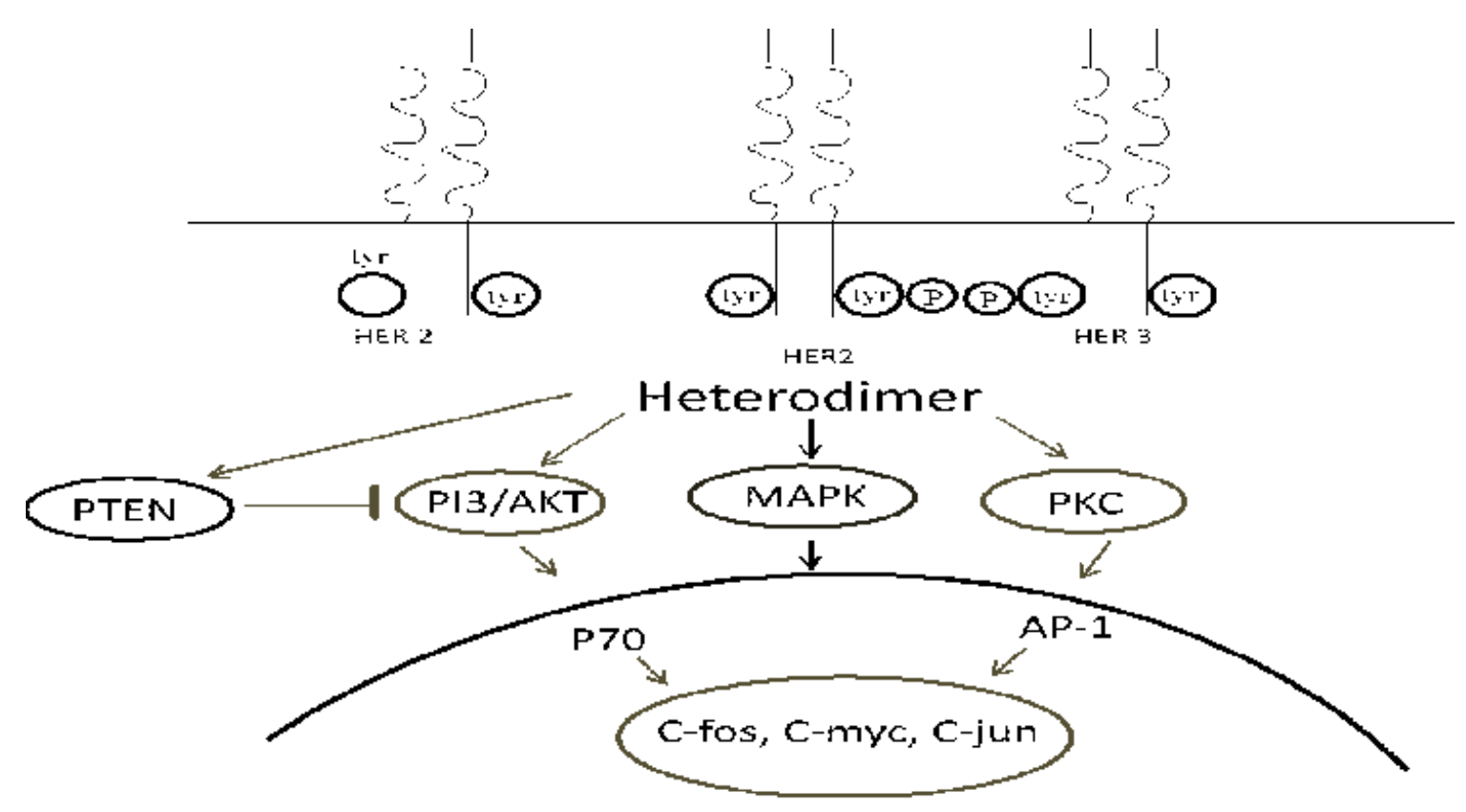

Figure 2

Figure (2): Schematic representation of HER2 signalling mechanism. Receptor tyrosine kinases cause dimerization of HER 2 by phophorylating at tyrosine residues which further activate various downstreaming pathways resulting in cancer..This heterodimerisation activate PI3/AKT,MAPK,PTEN and PKC pathways which further activate downstream signalling molecules P70 and AP-1 that in turn activates proto-oncogenes $\mathrm{c}-\mathrm{fos}, \mathrm{c}-\mathrm{myc}$ and c-jun involved in cell proliferation. However PTEN has antagonistic effects on thePI3/AKT pathway.

FOXP3 prevents metastasis and inhibits angiogenesis in breast carcinoma cells: The nuclear expression levels of the protein (Foxp3) were evaluated in the cell line of breast carcinoma. Among these cell lines, S.K.B.R-3 \& M.D.A-M.B-231, expressed relatively lower Foxp3 level,while T47D and M.D.AM.B-361, expressed relatively higher Foxp3 levels. Up regulation of Foxp 3 in S.K.B.R-3 and M.D.A-M.B-231 cells decreased the breast $\mathrm{Ca}$ cellinvasion capacity, whereas, Foxp3-siRNA mediated silencing in T47D and M.D.A-M.B-361 cell had about two fold elevation in the rate of tumor invasion in comparison with the control group cells. The results suggested that Foxp3 overexpression has an inhibitionimpact on breast $\mathrm{Ca}$ cellsinvasions \& adhesions, while Foxp3 silencings possesses an opposite impact. This could be due to the gene expression change viz. C.D.44 R.O.C.K2, E.C.M.1, D.L.G.A.P.5 and Serpine1 associated with cellular movement. Downregulation of these genes, particularly CD44, was most significant in reducing BC invasion and metastasis. C.D.44, the hyaluronic acid cell surface receptor is an adhesion molecule which has a long standing correlation with breast $\mathrm{Ca}$ cell invasions $\&$ metastasis ${ }^{[61-62]}$. Foxp3 mediated its inhibitory effect on breast $\mathrm{Ca}$ metastasis vialinkage with the promoters of gene encoding C.D.44 thereby inhibiting expression of breast $\mathrm{Ca}$ cells ${ }^{[63]}$. An inverselyrelation between CD44 expression and foxp3 reveals that FOXP3 inhibits metastasis via downregulation of CD44 expression ${ }^{\text {[64]. }}$ Chemokine CXCL12 and its receptor CXCR4 (CXCL12/ CXCR4) play amain role in regulating malignant cell growth, invasion, metastasis and secretion. Foxp3 regulates CXCL12/CXCR4 expression demonstrating an inverse correlation between Foxp3 and CXCL12/ CXCR4. Foxp3 showed low mRNA and protein levels in MCF-7 and MDA-MB-231 BC cell lines compared to normal breast epithelial cells. However, stable Foxp3 over expression in MDA-MB-231 cells led toreduced CXCR4, ErbB2/HER2, SKP20 and c-MYC expression. 
Thus, Foxp3 regulates breast $\mathrm{Ca}$ metastasis by downregulating the expression of some metastasis-related molecule including C.D.44 and C.X.C.R. $4^{[65] \text {. }}$

In addition to its inhibitory role in metastasis, fox p3 has also been implicated to inhibit VEGF- mediated angiogenesis. It was observed that ectopic expressions of Foxp3 in M.C.F-7, T47D and M.D.A-M.B- 231 cell lines downregulates VEGF expression and that silencing endogenous FOXP3 by shRNA upregulated VEGF expression at both the mRNA and protein levels. High Foxp3 expression was a protective factor for breast cancer survival, while high VEGF expression enhances breast cancer survival (Fig. 3). Foxp3-positive samples also showed a lower blood vessel density when compared with nuclear Foxp3- negative samples. These data hypothesize that Foxp3 is negatively related to angiogenesis in breast $\mathrm{Ca}$. Foxp3 inhibits breast $\mathrm{Ca}$ angiogenesis invitro and in vivo. This foxp3 mediated downregulation of VEGF took place by a direct interaction of forkhead-binding motifs of Foxp3 with the VEGF promoter $1.2 \mathrm{~kb}$ upstream of transcription start site, thus inhibiting VEGF promoter transcription and activity ${ }^{[66]}$.

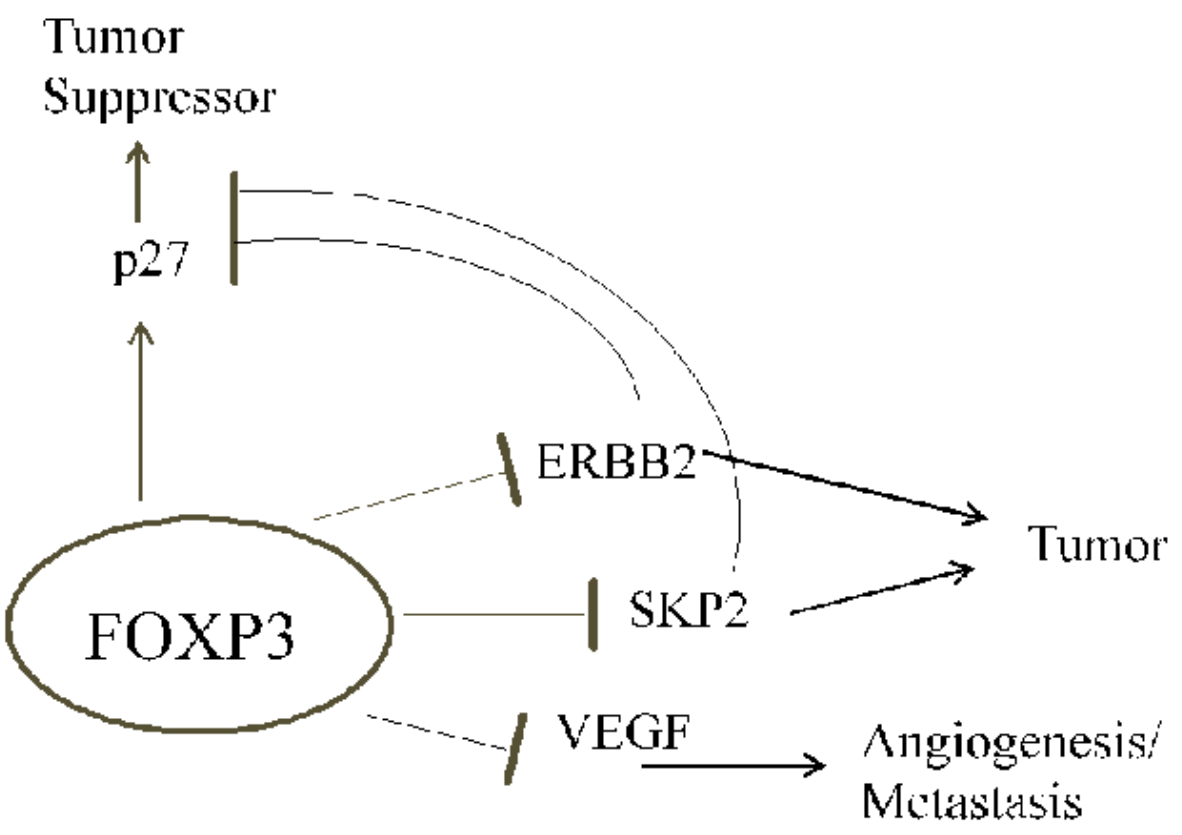

Figure 3

Figure (3): Schematic representation of role of Foxp3 as a inhibitor of oncogenes ERBB2 and SKP2 which cause breast cancer. Foxp3 also inhibits VEGF which is an important factor responsible for angiogenesis and metastasis to distant organs. Contrary to these Foxp3 maintains the expression of p27 a cell cycle inhibitor gene, thus exerting tumor suppressive function. Both ERBB2 and SKP2 inhibit expression of p27.

Therapies targeting FOXP3: In immunotherapy, the major hurdle is the T cell expressing Foxp3. Foxp3 is an intracellular transcriptional factor. It has been observed that the regulatory $\mathrm{T}$ cells elevate the breast cancer by suppressing the antitumor immunity. According to the research work carried out by Takeuchi, the tumors which are not recognized earlier by regulatory $\mathrm{T}$ cells triggers a strong antitumor response resulting in the blockage of several immune checkpoints. Thus, in order to elevate the efficacy of vaccines, Foxp3 expressing T regulatory cells are needed to be suppressed ${ }^{[67] .}$

Antigen, antibody, peptide or protein: In their study, Devi and Nath prepared novel vaccines containing synthetic mRNA encoding Foxp3 antigen and delivered them into dendritic cells. Upon transfection or pulsation the peptides of tumor antigens, total protein or total mRNA isolated from tumor cell, DNA encoding specific tumor antigens or synthetic tumor-antigenencoding mRNA entered into dendritic cells. These tumor antigen-associated dendritic cells resulted in 
subsequent generation of mature dendritic cells, which could efficiently elicit tumor antigen specific cytotoxic $\mathrm{T}$ lymphocytes fundamental in destruction of cancer cells $^{[68]}$.

A synthetic peptide P60 and antitumor DC vaccines were used to treat mice bearing LM3 and 4T1 tumors. P60 monotherapy was shown to inhibit tumor growth in immunocompetent in addition tothe immuno-compromised animals, primarily by inhibiting the secretion of immune-supressive cytokine IL-10 secretion in FOxp3 expressing breast carcinoma cells. Furthermore, combined treatment with antitumor dendritic cell vaccines and P60 increased the therapeutic efficacy of these vaccine in experimental models ${ }^{[69]}$.

\section{Conclusion}

The important role of FOXP3 as a tumor suppressor is evident by the fact that it not only represses HER2 and SKP2 breast cancer oncogenes, but also inhibits VEGF a pivotal player causing in angiogenesis and metastasis. However, it has totally contrary role in tumor milieu where its presence on the tumor infiltrating Treg cells results in suppression of anti-tumor response. As such patients initially respond to chemotherapeutic drugs develop resistance over a period of time. Patients who have Her- $2^{+}$over expressing breast cancer with primarily response to gradual trastuzumab acquired resistance. Foxp3 mediated inhibition of Her2 signaling mechanism has been extensively studied to identify additional targets to design effective treatmentsto those patients with drug-resistant $\mathrm{Her} 2^{+}$breast $\mathrm{Ca}$ patients. Studies have also shown that Foxp3 inhibition in Her2 ${ }^{+}$ overexpressing $\mathrm{BC}$ could be correlated with better prognosis and overall and relapse free survival. Thus, Foxp3 can be envisaged as a pivotal target whose down regulation in tandem with other breast cancer targeted therapies can have astounding effects in $\mathrm{BC}$ patients. A synergistic inhibition of Foxp3 in tumor milieu and anti-HER2 therapy on similar lines with anti-HER-2 and anti-VEGF therapy in HER2-positive breast carcinoma can be developed. Foxp3 can be envisaged as an important future candidate in designing an effective therapy against HER2- overexpression particularly drug resistant HER2- overexpressing breast cancer.

\section{Conflict of Interest: None}

\section{Source of Findings: None}

Ethical Clearance: None

\section{References}

1. Sjogren, S.; Inganas, M.; Lindgren, A.; Holmberg, L, ; Bergh, J. Prognostic and predictive value of c-erbB-2 overexpression in primary breast cancer, alone and in combination with other prognostic markers. J.Clin.Oncol., 1998, 16(2), 462-469.

2. Owens, M.A.; Horten, B.C.; Da Silva, M.M. HER2 amplification ratios by fluorescence in situ hybridization and correlation with immunohistochemistry in a cohort of 6556 breast cancer tissues. Clin. Breast. Cancer., 2004, 5(1), 63-69.

3. Seki, N.; Toh, U.; Hattori, S.; Kawahara, A.; Yamaguchi, T.; Koura, K.; Takahashi, R.; Otsuka, H.; Takahashi, H.; Iwakuma, N.; Nakagawa, S.; Fujii, T.; Sasada, T.; Yamaguchi, R.; Yano, H.; Shirouzu, K.; Kage, M.FOXP3 expression in tumor cells and tumor-infiltrating lymphocytes is associated with breast cancer prognosis. Mol.Clin. Oncol., 2013, 1(4), 625-632.

4. Graus-Porta, D.; Beerli, R.R.; Daly, J.M.; Hynes, N.E. ErbB-2, the preferred heterodimerization partner of all ErbB receptors, is a mediator of lateral signaling. EMBO., 1997, 16(7), 1647-1655

5. Yakes, F.M.; Chinratanalab, W.; Ritter, C.A.; King, W.; Seelig, S.; Arteaga, C.L. Herceptin-induced inhibition of phosphatidylinositol-3 kinase and Akt Is required for antibody-mediated effects on p27, cyclin D1, and antitumor action. Cancer Res., 2002, 62(14), 4132-4141.

6. Newman, L.; Xia, W.; Yang, H.Y.; Sahin, A.; Bondy, M.; Lukmanji, F.; Hung, M.C.; Lee, M.H. Correlation of p27 protein expression with HER2/neu expression in breast cancer. Mol.Carcinog., 2001, 30(3), 169-175.

7. Paplomata, E.; O'Regan, R. The PI3K/AKT/ mTOR pathway in breast cancer: targets, trials and biomarkers. Ther. Adv. Med. Oncol., 2014, 6(4), 154-166.

8. Hoffmann, C.; Mao, X.; Brown-Clay, J.; Moreau, F.; Al Absi, A.; Wurzer, H.; Sousa, B.; Schmitt, F.; Janji, B.; Berchem, G.; Thomas, C. Hypoxia promotes breast cancer cell invasion through HIF$1 \alpha$-mediated up-regulation of the invadopodial actin bundling protein CSRP2. Scientific Reports, 2018, 8:10191.

9. Le, X.F.; Mao, W.; Lu, C.; Thornton, A.; Heymach, J.V.; Sood, A.K.; Bast, R.C. Jr. Specific blockade 
of VEGF and HER2 pathways results in greater growth inhibition of breast cancer xenografts that overexpress HER2. Cell Cycle, 2008, 7(23), 37473758.

10. du Manoir, J.M.; Francia, G.; Man, S.; Mossoba, M.; Medin, J.A.; Viloria-Petit, A.; Hicklin, D.J.; Emmenegger, U.; Kerbel, R.S.Strategies for delaying or treating in vivo acquired resistance to trastuzumab in human breast cancer xenografts. Clin. Cancer Res., 2006, 12(3 pt 1), 904-916.

11. Rugo, H.S.; Jo Chien, A.; Franco, S.X.; Stopeck, A.T.; Glencer, A.; Lahiri, S.; Arbushites, M.C.; Scott, J.; Park, J.W.; Hudis, C.; Nulsen, B.; Dickler, M.N. A phase II study of lapatinib and bevacizumab as treatment for HER2-overexpressing metastatic breast cancer. Breast Cancer Res. Treat., 2012, 134(1), 13-20.

12. Miller, K.; Wang, M.; Gralow, J.; Dickler, M.; Cobleigh, M.; Perez, E.A.; Shenkier, T.; Cella, D.; Davidson, N.E. Paclitaxel plus bevacizumab versus paclitaxel alone for metastatic breast cancer. N. Engl. J. Med., 2007, 357(26), 2666-2676.

13. Baselgaetal., Lapatinib with trastuzumab for HER2- positive early breast cancer (NeoALTTO): a randomised, open-label, multicentre, phase 3 trial. Lancet, 2012, 379(9816), 633-640.

14. Falchook, G.S.; Moulder, S.L.; Wheler, J.J.; Jiang, Y.; Bastida, C.C.; Kurzrock, R. Dual HER2 inhibition in combination with anti-VEGF treatment is active in heavily pretreated HER2-positive breast cancer. Ann.Oncol., 2013, 24(12), 3004-3011.

15. Li, X.; Shen, L.; Zhang, J.; Su, J.; Shen, L.; Liu, X.; Han, H.; Han, W.; Yao, L. Degradation of HER2 by Cbl-based chimeric ubiquitin ligases. Cancer Res., 2007, 67(18), 8716-8724.

16. Eroglu, Z.; Tagawa, T.; Somlo, G.Human Epidermal Growth Factor Receptor FamilyTargeted Therapies in the Treatment of HER2Overexpressing Breast Cancer. Oncologist, 2014, 19(2), 135-150.

17. Eichhorn, P.J.; Gili, M.; Scaltriti, M.; Serra, V.; Guzman, M.; Nijkamp, W.; Beijersbergen, R.L.; Valero, V.; Seoane, J.; Bernards, R.; Baselga, J.PI3K Hyperactivation Results in Lapatinib Resistance that is Reversed by the mTOR/PI3K Inhibitor NVP-BEZ235. Cancer Res., 2008, 68(22), 9221-9230.
18. Xu, Z.Q.; Zhang, Y.; Li, N.; Liu, P.J.; Gao, L.; Gao, X.; Tie, X.J. Efficacy and safety of lapatinib and trastuzumab for HER2-positive breast cancer: a systematic review and meta-analysis of randomised controlled trials. BMJ. Open, 2017, 7(3):e013053.

19. Chung, C.; Lam, M.S. Pertuzumab for the treatment of human epidermal growth factor receptor type 2-positive metastatic breast cancer. Am. J. Health Syst. Pharm., 2013, 70(18), 1579-1587.

20. Swain etal., trastuzumab, and docetaxel for HER2positive metastatic breast cancer (CLEOPATRA study): overall survival results from a randomised, double-blind, placebo-controlled, phase 3 study. Lancet Oncol., 2013, 14(6), 461-471.

21. Chen, W.; He, J.; Song, S.; Wang, M.; Wu, H.; Wang, X. Efficacy of TCH/TEC neoadjuvant chemotherapy for the treatment of HER-2overexpressing breast cancer. Oncol. Lett., 2015, 9(4), 1922-1926.

22. Huszno, J. and Nowara, E. Current therapeutic strategies of anti-HER2 treatment in advanced breast cancer patients. Contemp.Oncol (Pozn)., 2016, 20(1), 1-7.

23. von Minckwitz, G.; Huang, C.S.; Mano, M.S.; Loibl, S.; Mamounas, E.P.; Untch, M.; Wolmark, N.; Rastogi, P.; Schneeweiss, A. TrastuzumabEmtansine for Residual Invasive HER2-Positive Breast Cancer. N. Engl. J. Med., 2019, 380(7), 617-628.

24. Siddique, A.B.; Ebrahim, H.Y.; Akl, M.R.; Ayoub, N.M.; Goda, A.A.; Mohyeldin, M.M.; Nagumalli, S.K.; Hananeh, W.M.; Liu, Y.Y.; Meyer, S.A.; El Sayed, K.A.(-)-Oleocanthal Combined with Lapatinib Treatment Synergized against HER2 Positive Breast Cancer In Vitro and In Vivo. Nutrients, 2019, 11(2), 412.

25. Brunkow, M.E.; Jeffery, E.W.; Hjerrild, K.A.; Paeper, B.; Clark, L.B.; Yasayko, S.A.; Wilkinson, J.E.; Galas, D.; Ziegler, S.F.; Ramsdell, F. Disruption of a new forkhead/winged-helixprotein, scurfin, results in the fatal lymphoproliferative disorder of the scurfy mouse. Nat. Genet., 2001, 27(1), 68-73.

26. Lozano, T.; Casares, N.; Lasarte, J.J. Searching for the Achilles heel of FOXP3. Front.Oncol., 2013, 3:294.

27. Chatila etal., encoding a forkhead-related protein, is mutated in $\mathrm{X}$ - linked autoimmunity-allergic 
disregulation syndrome. J.Clin. Invest., 2000, 106(12), R75-81.

28. Wildin etal., X-linked neonatal diabetes mellitus, enteropathy and endocrinopathy syndrome is the human equivalent of mouse scurfy. Nat. Genet., 2001, 27(1), 18-20.

29. Sakaguchi, S.; Yamaguchi, T.; Nomura, T.; Ono, M. Regulatory T cells and immune tolerance. Cell, 2008, 133(5), 775-787.

30. Shimizu, J.; Yamazaki, S.; Sakaguchi, S. Induction of tumor immunity by removing CD25+CD4+ T cells: a common basis between tumor immunity and autoimmunity. J.Immunol., 1999, 163(10), 5211-5218.

31. Yamaguchi, T. and Sakaguchi, S. Regulatory T cells in immune surveillance and treatment of cancer. Semin. Cancer Biol., 2006, 16(2), 115-123.

32. Ono etal., .Foxp3 controls regulatory T-cell function by interacting with AML1/Runx 1. Nature, 2007, 446(7136), 685-689.

33. Wu, Y.; Borde, M.; Heissmeyer, V.; Feuerer, M.; Lapan, A.D.; Stroud, J.C.; Bates, D.L.; Guo, L.; Han, A.; Ziegler, S.F.; Mathis, D.; Benoist, C.; Chen, L.; Rao, A. FOXP3 controls regulatory $\mathrm{T}$ cell function through cooperation with NFAT. Cell, 2006, 126(2), 375-387.

34. Pandiyan etal., CD4+CD25+Foxp3+ regulatory $\mathrm{T}$ cells induce cytokine deprivation-mediated apoptosis of effector CD4+ T cells. Nat.Immunol., 2007, 8(12), 1353-1362.

35. Schubertetal., Autosomal dominant immune dysregulation syndrome in humans with CTLA4 mutations. Nat. Med., 2014, 20(12), 1410-1416.

36. Kuehnetal., , Immune dysregulation in human subjects with heterozygous germline mutations in CTLA4. Science, 2014, 345(6204), 1623-1627.

37. Takahashi etal., Immunologic self-tolerance maintained by $\mathrm{CD} 25+\mathrm{CD} 4+$ regulatory $\mathrm{T}$ cells constitutively expressing cytotoxic $\mathrm{T}$ lymphocyteassociated antigen 4. J. Exp. Med., 2000, 192(2), 303-310.

38. Qureshi etal., Trans-endocytosis of CD80 and CD86: a molecular basis for the cell-extrinsic function of CTLA-4. Science, 2011, 332(6029), 600-603.

39. Yamaguchi, T.; Kishi, A.; Osaki, M.; Morikawa, H.; Prieto-Martin, P.; Wing, K.; Saito, T.; Sakaguchi, S.
Construction of self-recognizing regulatory $\mathrm{T}$ cells from conventional $\mathrm{T}$ cells by controlling CTLA-4 and IL-2 expression. Proc. Natl. Acad. Sci. USA., 2013, 110(23), E2116-E2125.

40. Liu, Z.; Gerner, M.Y.; Van Panhuys, N.; Levine, A.G.; Rudensky, A.Y.; Germain, R.N. Immune homeostasis enforced by co-localized effector and regulatory T cells. Nature, 2015, 528(7581), 225230.

41. Wolf, A.M.; Wolf, D.; Steurer, M.; Gast, G.; Gunsilius, E.; Grubeck-Loebenstein, B. Increase of regulatory $\mathrm{T}$ cells in the peripheral blood of cancer patients. Clin. Cancer Res. 2003, 9(2), 606-612.

42. Ormandy, L.A.; Hillemann, T.; Wedemeyer, H.; Manns, M.P.; Greten, T.F.; Korangy, F. Increased populations of regulatory $\mathrm{T}$ cells in peripheral blood of patients with hepatocellular carcinoma. Cancer Res., 2005, 65(6), 2457-2464.

43. Schaefer, C.; Kim, G.G.; Albers, A.; Hoermann, K.; Myers, E.N.; Whiteside, T.L. Characteristics of $\mathrm{CD} 4+\mathrm{CD} 25+$ regulatory $\mathrm{T}$ cells in the peripheral circulation of patients with head and neck cancer. Br. J. Cancer, 2005, 92(5), 913-920.

44. Liyanage, U.K.; Moore, T.T.; Joo, H.G.; Tanaka, Y.; Herrmann, V.; Doherty, G.; Drebin, J.A.; Strasberg, S.M.; Eberlein, T.J.; Goedegebuure, P.S.; Linehan, D.C. Prevalence of regulatory T cells is increased in peripheral blood and tumor microenvironment of patients with pancreas or breast adenocarcinoma. J. Immunol., 2002, 169(5), 2756-2761.

45. Hiraoka, N.; Onozato, K.; Kosuge, T.; Hirohashi, S. Prevalence of FOXP3 + regulatory $\mathrm{T}$ cells increases during the progression of pancreatic ductal adenocarcinoma and its premalignant lesions. Clin. Cancer Res., 2006, 12(18), 5423-5434.

46. Satoetal., Intraepithelial CD8+ tumor-infiltrating lymphocytes and a high CD8+/regulatory $\mathrm{T}$ cell ratio are associated with favorable prognosis in ovarian cancer. Proc. Natl. Acad. Sci. USA., 2005, 102(51), 18538-18543.

47. Nishikawa, H.; Sakaguchi, S. Regulatory T cells in tumor immunity. Int. J. Cancer, 2010, 127(4), 759767.

48. Bates, G.J.; Fox, S.B.; Han, C.; Leek, R.D.; Garcia, J.F.; Harris, A.L.; Banham, A.H. Quantification of regulatory $\mathrm{T}$ cells enables the identification of highrisk breast cancer patients and those at risk of late relapse. J.Clin.Oncol., 2006, 24(34), 5373-5380. 
49. Sasada, T.; Kimura, M.; Yoshida, Y.; Kanai, M.; Takabayashi, A. CD4+CD25+ regulatory $\mathrm{T}$ cells in patients with gastrointestinal malignancies: possible involvement of regulatory $\mathrm{T}$ cells in disease progression. Cancer, 2003, 98(5), 10891099.

50. Curiel etal., Specific recruitment of regulatory $\mathrm{T}$ cells in ovarian carcinoma fosters immune privilege and predicts reduced survival. Nat. Med. 2004, 10(9), 942-949.

51. Shang, B.; Liu, Y.; Jiang, S.; Liu, Y. Prognostic value of tumor-infiltrating FoxP3+ regulatory $\mathrm{T}$ cells in cancers: a systematic review and metaanalysis. Sci. Rep., 2015, 5:15179.

52. Perez, S.A.; Karamouzis, M.V.; Skarlos, D.V.; Ardavanis, A.; Sotiriadou, N.N.; Iliopoulou, E.G.; Salagianni, M.L.; Orphanos, G.; Baxevanis, C.N.; Rigatos, G.; Papamichail, M. CD4+CD25+ Regulatory T-Cell Frequency in HER-2/neu (HER)-Positive and HER-Negative AdvancedStage Breast Cancer Patients. Clin. Cancer Res., 2007, 13(9), 2714-2721.

53. Knoechel, B.;Lohr, J.; Kahn, E.; Bluestone, J.A.; Abbas, A.K. Sequential development of interleukin 2-dependent effector and regulatory $\mathrm{T}$ cells in response to endogenoussystemic antigen. J. Exp. Med., 2005, 202(10), 1375-1386.

54. Lohr, J.;Knoechel, B.; Abbas, A.K. Regulatory T cells in the periphery. Immunol Rev., 2006, 212, 149-62.

55. Schuurhuis, D.H.; van Montfoort, N.;IoanFacsinay, A.; Jiawan, R.; Camps M.; Nouta, J.; Melief, C.J.; Verbeek, J.S.; Ossendorp, F. Immune complex-loaded dendritic cells are superior to soluble immune complexes as antitumor vaccine. J.Immunol., 2006, 176(8), 4573-4580.

56. Bennett, C.L.; Christie, J.;Ramsdell, F.;Brunkow, M.E.; Ferguson, P.J.;Whitesell, L.; Kelly, T.E.;Saulsbury, F.T.; Chance, P.F.; Ochs, H.D. The immune dysregulation, polyendocrinopathy, enteropathy, X-linked syndrome (IPEX) is caused by mutations of FOXP3. Nat. Genet., 2001, 27(1), 20-21.

57. Brunkowetal., Disruption of a new forkhead/ winged-helix protein, scurfin, results in the fatal lymphoproliferative disorder of the scurfy mouse. Nat. Genet., 2001, 27(1), 68-73.
58. Chatilaetal., encoding a fork head-related protein, is mutated in X-linked autoimmunity-allergic disregulation syndrome. J.Clin. Invest., 2000, 106(12), R75-81.

59. Wildinetal., X-linked neonatal diabetes mellitus, enteropathy and endocrinopathy syndrome is the human equivalent of mouse scurfy. Nat. Genet., 2001, 27(1), 18-20.

60. Zuoetal., FOXP3 is an X-linked breast cancer suppressor gene and an important repressor of the HER-2/ErbB2 oncogene. Cell, 2007, 129(7), 12751286.

61. Hill, A.; McFarlane, S.; Mulligan, K.; Gillespie, H.; Draffin, JE.; Trimble, A.; Ouhtit, A.; Johnston, P.G.; Harkin, D.P.; McCormick, D.; Waugh, D.J.Cortactin underpins CD44-promoted invasion and adhesion of breast cancer cells to bone marrow endothelial cells. Oncogene, 2006, 25(45), 60796091.

62. Ouhtit, A.; Abd Elmageed, Z.Y.; Abdraboh, M.E.; Lioe, T.F.; Raj, M.H. In vivo evidence for the role of CD44s in promoting breast cancer metastasis to the liver. Am. J. Pathol., 2007, 171(6), 2033-2039.

63. Abraham, B.K.; Fritz, P.; McClellan, M.; Hauptvogel, P.; Athelogou, M.; Brauch, H. Prevalence of CD441/CD24-/low cells in breast cancer may not be associated with clinical outcome but may favor distant metastasis. Clin. Cancer Res., 2005, 11(3), 1154-1159.

64. Zhang etal., FOXP3 suppresses breast cancer metastasis through downregulation of CD44. Int. J. Cancer, 2015, 137(6), 1279-1290.

65. Douglass, S.; Meeson, A.P.; Overbeck-Zubrzycka, D.; Brain, J.G.; Bennett, M.R.; Lamb, C.A.; Lennard, T.W.; Browell, D.; Ali, S.; Kirby, J.A. Breast cancer metastasis: demonstration that FOXP3 regulates CXCR4 expression and the response to CXCL12. J.Pathol., 2014, 234(1), 7485.

66. Li, X.; Gao, Y.; Li, J.; Zhang, K.; Han, J.; Li, W.; Hao, Q.; Zhang, W.; Wang, S.; Zeng, C.; Zhang, W.; Zhang, Y.; Li, M.; Zhang, C. FOXP3 inhibits angiogenesis by downregulating VEGF in breast cancer. Cell Death Dis., 2018, 9(7), 744

67. Takeuchi, Y.; Nishikawa, H. Roles of regulatory $\mathrm{T}$ cells in cancer immunity. Int.Immunol., 2016, 28(8), 401-419. 
68. Devi, G.R.; Nath, S. Delivery of Synthetic mRNA Encoding FOXP3 Antigen into Dendritic Cells for Inflammatory Breast Cancer Immunotherapy. Method Mol. Biol., 2016, 1428, 231-243.
69. Moreno Ayala, M.A.; Gottardo, M.F.; Imsen, M.; Asad, A.S.; Bal de Kier Joffé, E.; Casares, N.; Lasarte, J.J.; Seilicovich, A.; Candolfi, M.; Therapeutic blockade of Foxp3 in experimental breast cancer models. Breast Cancer Res. Treat., 2017, 166(2), 393-405. 